\title{
Establishing teams aiming to provide culturally safe maternity care for Indigenous families
}

\author{
Sophie Hickey ${ }^{\mathrm{a}, \mathrm{b}, *}$, Sue Kildea ${ }^{\mathrm{b}}$, Katrina Couchman ${ }^{\mathrm{c}}$, Kristie Watego-Ivory ${ }^{\mathrm{c}}$, \\ Roianne West ${ }^{\mathrm{d}}$, Sue Kruske ${ }^{\mathrm{c}}$, Renee Blackman ${ }^{\mathrm{e}}$, Shannon Watego ${ }^{\mathrm{f}}$, Yvette L. Roe ${ }^{\mathrm{b}}$ \\ a Mater Research Institute-University of Queensland, Australia \\ ${ }^{\mathrm{b}}$ Charles Darwin University, Australia \\ ${ }^{\mathrm{c}}$ The Institute for Urban Indigenous Health, Australia \\ ${ }^{\mathrm{d}}$ Griffith University, Australia \\ e Aboriginal and Torres Strait Islander Community Health Service, Brisbane Ltd, Australia \\ ${ }^{\mathrm{f}}$ Mater Misericordiae Limited, Australia
}

\section{A R T I C L E I N F O}

\section{Article history:}

Received 12 April 2019

Received in revised form 25 June 2019

Accepted 27 June 2019

Available online $\mathrm{xxx}$

\section{Keywords:}

Cultural safety

Health service research

Health workforce

Indigenous health

Participatory action research

Midwifery

\begin{abstract}
A B S T R A C T
Problem: It is not well known how to prepare new multidisciplinary teams aiming to provide culturally safe maternity care for Aboriginal and Torres Strait Islander families in an urban setting.

Background: National policies recommend increasing the Aboriginal and Torres Strait Islander workforce and cultural competencies of the non-Indigenous workforce as key drivers of culturally safe care. Question: What are the key learnings from staff experiences establishing multidisciplinary teams aiming to provide culturally safe maternity care that aims to privilege Indigenous ways of knowing, being and doing? Methods: As part of a larger participatory action research project, semi-structured qualitative interviews were conducted December 2014-April 2015 with 21 Aboriginal and Torres Strait Islander and nonIndigenous healthcare staff. Thematic analysis was used to identify learnings for practice.

Findings: Four key learnings were identified for forming new teams aiming to provide culturally safe care: (a) having a shared understanding of what characterises cultural safety in the local program context; (b) understanding and valuing different roles and knowledges people bring to the team; (c) acknowledging the influence of race and culture on staff behaviour; and (d) acting on individual and organisational responsibilities for continuous improvement towards cultural safety.

Discussion: We present recommendations from our participatory action research approach to respond to these learnings in practice.

Conclusion: A deliberate workforce investment at the early stages of team development is crucial when aiming to provide culturally safe maternity care that can respond to the unique needs of Aboriginal and Torres Strait Islander women and families.
\end{abstract}

(C) 2019 Australian College of Midwives. Published by Elsevier Ltd. All rights reserved.

\section{Statement of significance}

\section{Problem or issue}

A clinically and culturally safe health workforce is essential to accelerating much-needed health gains for Aboriginal and Torres Strait Islander people. It is not well known how to establish new multidisciplinary teams aiming to provide culturally safe maternity care for Aboriginal and Torres Strait Islander women and families in an urban setting.

What is already known

Over three decades of policy recommendations have been to increase the Indigenous maternity workforce and improve cultural competencies of the wider workforce. However, there remains little evidence in what works to support nonIndigenous staff aspiring to become culturally safe in practice.

\section{What this paper adds}

We provide recommendations for investment in the workforce at the initial stages of team development. These privilege Indigenous ways of knowing, being and doing as a crucial component of developing a maternity service that aims to be culturally safe and responds to the clinical and cultural needs of Aboriginal and Torres Strait Islander families.

\footnotetext{
* Corresponding author at: Mater Research, Level 2 Aubigny Place, Raymond Tce, South Brisbane QLD 4101, Australia.

E-mail address: sophie.hickey@mater.uq.edu.au (S. Hickey).
} 


\section{Background}

A clinically and culturally safe health workforce is essential to accelerating much-needed health gains for Aboriginal and Torres Strait Islander people, the Indigenous peoples of Australia. ${ }^{1-3}$ To achieve this, existing literature and policy recommends (a) increasing the Aboriginal and Torres Strait Islander health workforce and (b) providing cultural competency training to the non-Indigenous health staff ${ }^{2,4,5}$ (see Box 1 for definitions of cultural safety and cultural competency). However, there is limited evidence on the most effective ways to 'form' and 'storm'5 (see Box 2) new multidisciplinary teams of Indigenous and non-Indigenous staff working in new services that aim to privilege Indigenous ways of knowing, being and doing. ${ }^{6}$ The National Aboriginal Strategy ${ }^{1}$ clearly defines a holistic approach to health and wellbeing informed by Aboriginal and Torres Strait Islanders ways of knowing (epistemology), being (ontology) and doing (axiology): 'Health is ... not just the physical well-being of the individual but the social, emotional, and cultural well-being of the whole community. This is a whole-of-life view and it also includes the cyclical concept of life-death-life' ( $p$. x). This has been the foundation of healthy communities on this continent for millennia as the oldest living cultures on Earth. Ongoing impacts of colonisation, policies of assimilation, and the undermining of Aboriginal and Torres Strait Islander selfdetermination ${ }^{7}$ have marginalised Indigenous knowledges and

Box 1. Cultural safety and cultural competence.

Cultural safety describes an environment that is safe; where there is no assault, challenge or denial of one's identity, of who they are and what they need..$^{12}$ Importantly, this is defined by the service user, and considers the influences of power imbalances and impacts of racism and colonisation. ${ }^{12}$ Cultural safety requires health professionals to critically reflect on their own practice so they can adapt to meet the needs of healthcare consumers. ${ }^{12}$ A culturally competent workforce is one that has adequate knowledge and skills to interact with clients in a culturally safe way. ${ }^{13}$

resulted in the current Australian maternity system failing to meet the needs of Aboriginal and Torres Strait Islander people, perpetuating inequitable health outcomes ${ }^{8,9}$. In Australia, the majority of births (97\%) take place in the hospital setting, ${ }^{8}$ with Aboriginal and Torres Strait Islander Community Controlled Health Organisations (ACCHOs) providing antenatal and

Box 2. Tuckman's ${ }^{14}$ stages of small group development.

Management literature describes 'forming' as the first stage of team formation: when new staff come together with limited common understanding of the team's aims, and individual roles and responsibilities. Next is 'storming', when team members try to establish boundaries and experience difficulties deciding common goals or ways of working. 'Norming' will see the team deciding on new rules of engagement, and individual roles and responsibilities become clearer, with common understanding of each person's contribution. 'Performing' teams are harmonious, have clarity of task, team loyalty and cohesion and creative problem-solving. Added at a later date, final stage 'adjorning' describes the end of a team lifecycle through separation. ${ }^{15}$ postnatal care, with none currently providing intrapartum care. Maternity staff work within a predominantly biomedical and fractured health system largely designed by the dominant cultural group for the dominant cultural group. Ideally, specialist Indigenous maternity services redesign should occur in partnership with Aboriginal and Torres Strait Islander women, children and families, ${ }^{10,11}$ incorporate Indigenous knowledges and cultural practices ${ }^{11}$ and embed the holistic definition of health and wellbeing ${ }^{1}$. This requires staff to acknowledge the impact of the dominant Western medical paradigms and rethink how to stay responsive to the needs of women and families - all the while navigating organisational pressures, establishing team culture, new roles and shared understandings of what it means to work towards a culturally safe service.

\subsection{Culturally safe maternity care and contested knowledge holders}

When teams of midwives and Indigenous health workers come together, with the aim to provide clinically and culturally safe maternity care for Aboriginal and Torres Strait Islander families, they bring together a mix of clinical midwifery knowledge and Indigenous cultural knowledges which are embedded in community knowledge. ${ }^{16,17}$ Midwifery philosophy and Indigenous healthcare philosophy align through the centring of respectful relationships to empower (Indigenous) women to self-determine their own birthing and parenting choices. ${ }^{16}$ Yet, Indigenous knowledges are seldom incorporated or privileged in Australian healthcare, rather Western Scientific knowledge remains the dominant knowledge system and is never truly systematically challenged. ${ }^{18}$ Beyond 'a simple intersection of two different and often contesting elements', ${ }^{18}$ it is at this interface where Indigenous and non-Indigenous people have greater opportunity to work together to generate new ways of understanding. ${ }^{18}$

Increasing the cultural competency of the health workforce is now recognised as a requirement for accreditation standards, ${ }^{19}$ professional standards ${ }^{20}$ and codes of conduct. ${ }^{21}$ However, there remains little evidence in what works to support non-Indigenous staff aspiring to become culturally safe in practice, with inconsistent training nationally. ${ }^{22-24}$ It can be problematic when Indigenous staff are informally or formally relied on as cultural educators, ${ }^{25}$ cultural brokers, or cultural mentors, ${ }^{26}$ beyond their scope of practice, expertise or remuneration. ${ }^{25,27,28}$ This can sometimes be a challenging or traumatic experience for Indigenous staff, ${ }^{25,28}$ despite a strong desire to change the attitudes and behaviours of non-Indigenous staff ${ }^{28}$. There remains limited health system supports for both Indigenous and non-Indigenous staff beyond their small specialised programs and teams.

Forming and norming such teams can be a vexed space and requires reflection on the social construction of race and ethnicity; ${ }^{29}$ something not routinely discussed explicitly in mainstream Australian workplaces. This includes identifying overt and subversive influences on how dominant groups perceive their culture as 'normal' and non-dominant groups as 'other', and power imbalances at play $^{30}$ - a central tenant of cultural safety. ${ }^{12}$ Whiteness refers to the process by which White non-Indigenous people are afforded normalised unearned privileges as the dominant group. ${ }^{31}$ Acknowledging whiteness makes visible the power imbalances that may occur in the healthcare context between Indigenous and non-Indigenous staff, where nonIndigenous people are often in roles of higher positional power and are supported by a health system that reinforces their dominant worldview, whose legitimacy remains largely unchallenged. Non-Indigenous people may have limited familiarity or experience working with Indigenous people in Australia; ${ }^{7}$ consequently, they may be unaware, unprepared or uncomfortable 


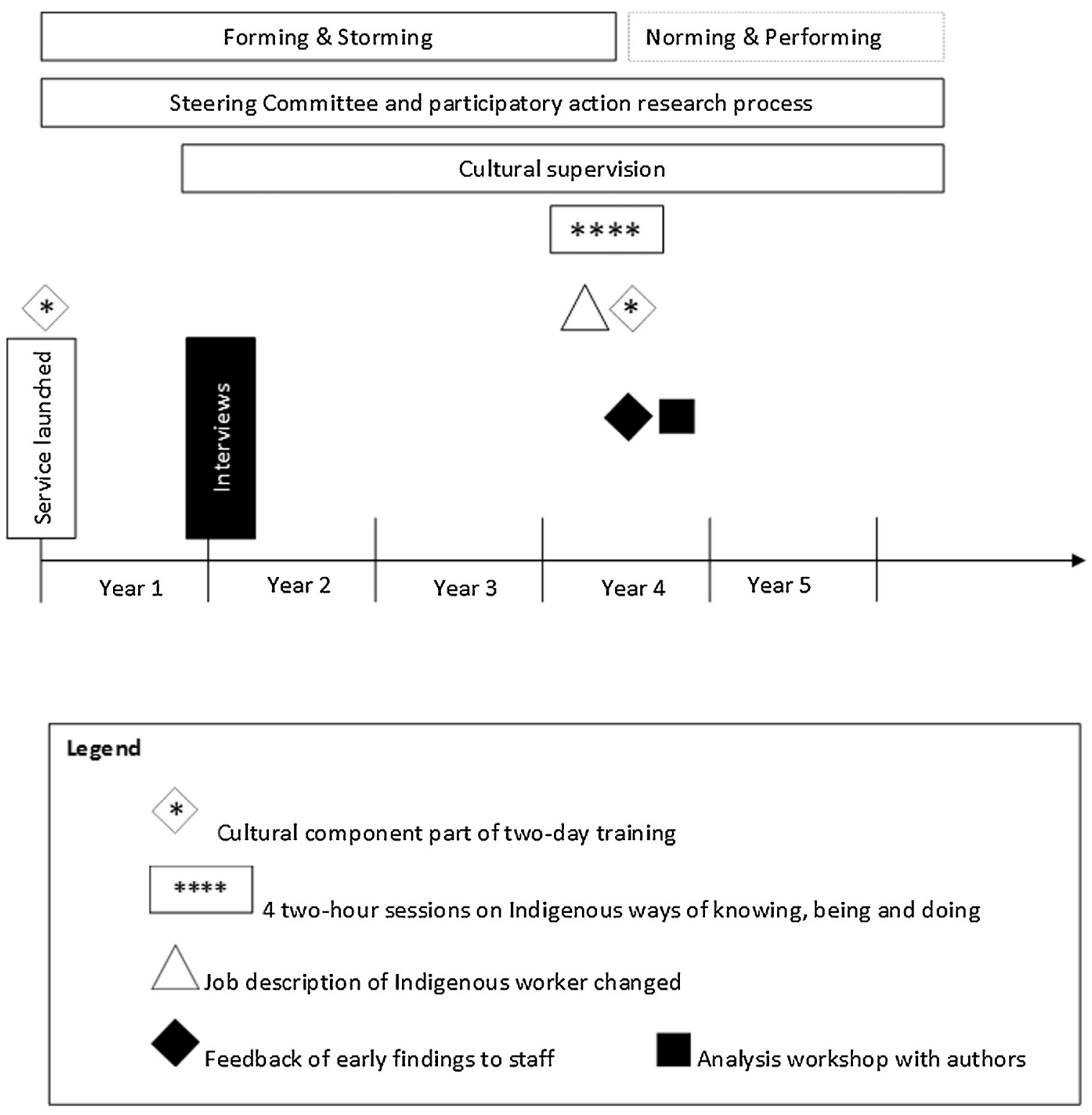

Fig. 1. Timeline of establishment of service, cultural safety training and research activities.

to think critically about issues facing Indigenous people such as racism, colonisation and intergenerational trauma. ${ }^{7,32}$ The term mainstream health service restates this embedded uncontested power imbalance. In contrast, Indigenous knowledge and community knowledge - crucial to culturally safe health service provision - are not afforded the same privileged position. ${ }^{17}$ This represents a system of institutionalised racism; independent of intent, individuals can reproduce acts of racial or cultural oppression. ${ }^{33}$ It is from this marginalised position that Indigenous people continue to call for greater accountability of the health system to address systematic issues of culturally unsafe care and, importantly, to incorporate Indigenous perspectives and worldviews in healthcare. ${ }^{1,34}$

For health managers and staff aiming to establish cohesive teams that provide culturally safe care, early formation that builds the culture of the team is a process that requires non-Indigenous and Indigenous staff to meet at this interface and generate new ways of working. To date, there has been little attention to how these multidisciplinary teams navigate these early stages of team formation.

\section{Aim}

This study aimed to identify key learnings for practice from staff perspectives and experiences of the first year establishing a new multidisciplinary team that aimed to provide culturally safe maternity care for Aboriginal and Torres Strait Islander women and families in an urban setting.

\section{Methods}

This study formed part of a broader participatory action research project $^{35}$ designed to improve maternity care for Aboriginal and Torres Strait Islander women and families in a large Australian city on the sovereign lands of the Yuggera and Turrbal people. This is home to the largest and fastest growing population of Aboriginal and Torres Strait Islander people in Australia. ${ }^{36}$ The project was initiated in response to a local community-identified need to improve clinical and cultural safety of the maternity service throughout the pregnancy, labour and birth and postnatal journey (more below and detailed elsewhere). ${ }^{37}$ Participatory action research is an 
approach that prioritises local perspectives, needs and knowledge through partnerships and collaborations with community and key stakeholders throughout the research process. ${ }^{38,39}$ Community and stakeholders are co-researchers, and data is pragmatically used to assess, respond, reflect then act with the aim of creating positive social change. ${ }^{38}$ This study had several mechanisms to enable the participatory action research approach (Fig. 1). These included an overarching Steering Committee that was established by the three partner organisations with members having joint responsibility for resource allocation, management decisions and overarching governance and met regularly. A monthly working group was established to troubleshoot local operational issues with middle management and frontline staff involved in service delivery, and the core research team met every two weeks ${ }^{40}$. Regular communication, reflection and action between these groups was enabled through the principle investigator of the larger study who was the Chair of all groups. This study aligned with national guidelines and principles for health research with Aboriginal and Torres Strait Islander people. ${ }^{41,42}$ Relevant Human Research Ethics Committee and Research Governance approvals were granted for this study by the hospital organisation (HREC/14/MHS/168).

\subsection{Study setting}

In this study setting, a partnership was formed between a large tertiary maternity hospital and two local Aboriginal Community Community Health Organisations with the aim to provide culturally safe continuity of care for women having Indigenous babies. The Steering Committee consisted of senior staff from the three organisations and provided overarching Indigenous governance for the project. At program inception, non-Indigenous midwives (not Indigenous identified positions) and Indigenous maternal and infant health workers (positions identified for Indigenous people) were employed by the hospital. Clinical and managerial support provided by the three partner organisations. The women accessing the new service received 24/7 caseload midwifery care during pregnancy to six weeks postnatally, with the Indigenous health workers and Indigenous liaison officers providing social-emotional, cultural and practical support during weekday business hours. Midwives commenced with an 8-week clinical orientation at the hospital. Staff involved in frontline service delivery attended orientation at each of the partner organisation and an initial two-day orientation at one of the ACCHOs that included a component about the social, historical and structural factors that impact the health of Aboriginal and/or Torres Strait Islander peoples. ${ }^{21}$ Joint clinical and cultural supervision to guide critical reflective practice ${ }^{20}$ was initiated by one of the ACCHOs for the midwives during the data collection period. As part of the participatory action research approach, findings were presented back to the frontline staff as part of a team building exercise and were used to frame the transition to the norming and performing stages of team development ${ }^{15}$. Findings were also fed back to the Steering Committee which led to changes being made to staff orientation, clinical and cultural supervision as well as off-site workshops (details in Discussion).

\subsection{Participants}

Participants for this study were purposefully selected: all staff involved in establishing the new partnership-based, multidisciplinary model of care were invited to participate in the study. Some had been redeployed from within the partner organisations, others were newly recruited. Roles of staff included: Chief Executive Officers, managers, frontline staff (clinicians, direct service delivery), and support staff (e.g. project coordinators, tutors, supervision facilitators). There was proportionate representation from the three partner organisations, role type and Indigenous status of participants (Table 1 ), with 21 of the 30 invited staff participating in the study. Participants were initially approached via email, with email or face-to-face follow-ups. Reasons for nonparticipation included participants busy work schedules, as well as other staff feeling as though they had not been involved sufficiently in the project at that time to be able to contribute anything useful, loss to follow-up, moved out of the role, and declined (no reason given).

\subsection{Informed consent and data collection}

This study draws from a dataset of qualitative interviews collected as part of the larger participatory action research project. Interviews were conducted after the first year establishing the new service between December 2014 and April 2015. This was undertaken by the third author, a non-Indigenous health professional on research placement with one of the ACCHOs who had no prior relationships with the participants. Indigenous oversight was provided by a senior Indigenous health researcher (last author) and reflexive field notes were taken throughout the data collection period. An interview guide of five question prompts was devised and pilot tested exploring perceptions and experiences of the partnership, its strengths and challenges, and opportunities for improvement. Participants were emailed the participant information and consent form when initially invited to participate in the study. Prior to commencing the interview, the interviewer verbally explained the project and assured participants that they would not be disadvantaged in any way by participating or not in the study. Once all questions were answered, written informed consent was freely given. Interviews were conducted in a private workplace setting (on the phone or in person) during work hours, with support from management. This comprised of 18 semi-structured qualitative interviews, ${ }^{43}$ comprising of 11 individual face-to-face interviews; five phone interviews; and two face-to-face group interviews with two and three participants. The group interviews ${ }^{44}$ were requested by the participants themselves, with members sharing similar role types and cultural backgrounds. Advantages of individual interviews included in-depth data collection from an individual's own perspective and increased privacy. Face-to-face interviews provided the interviewer with greater capacity to read body language and probe accordingly for deeper understanding and explanations of responses; recognising that communication is inherently a socio-cultural experience therefore is mediated by cultural backgrounds of interviewer and interviewees. ${ }^{45}$ Disadvantages of group interviews can include more conformity in view expressed, with increased reluctance to discuss sensitive topics and greater irrelevant discussion. ${ }^{44}$ However, group interviews provide the opportunity to interrogate how and why different groups think and feel about a topic, as well as saving time and money compared to individual interviews ${ }^{44}$. All interviews were audio-recorded with permission and ranged from 15 to 65 minutes.

Table 1

Interviewee sample characteristics.

\begin{tabular}{ll}
\hline Demographics & Consented \& participated $(\mathrm{n}=21)$ \\
\hline Indigenous status & \\
$\quad$ Indigenous & $10(48 \%)$ \\
Non-Indigenous & $11(52 \%)$ \\
& \\
Role & \\
Service delivery staff & $13(62 \%)$ \\
Managers & $8(38 \%)$ \\
\hline
\end{tabular}




\subsection{Data analysis}

Interviews were firstly transcribed by an external company, checked for accuracy by the first and third authors, with participants allocated pseudonyms. Aboriginal and Torres Strait Islander status of participants was retained, though role type and organisation has since been omitted to preserve confidentiality and anonymity of participants. Analysis was an iterative and reflective process, guided by the aim to generate relevant and practice-based recommendations. The first author used thematic analysis using an inductive approach to organise the data. ${ }^{46}$ The first author is non-Indigenous with experience in Indigenous health research. Emergent themes were verified by a senior Aboriginal researcher (last author). Next, preliminary themes were presented at a workshop with partnership frontline staff and to the Steering Committee. A subsequent workshop was held with a group of Indigenous midwifery academics and ACCHO managers within the research Investigators team to provide feedback and refinement of the themes and recommendations. Themes were reviewed and refined following consensus among the group who privileged Indigenous perspectives if/when needed. Quotations were selected to best illustrate themes, with attention to diverse cases.

\section{Findings}

Four key learnings were identified as important when forming new teams aiming to be culturally safe: (a) having a shared understanding of what characterises culturally safety in the local program context; (b) understanding and valuing different roles and knowledges people bring to the team; (c) acknowledging the influence of race and culture on staff behaviour; and (d) acting on individual and organisational responsibilities for continuous improvement towards cultural safety. Some differences in experiences and perspectives were found between Indigenous and non-Indigenous staff (see Fig. 2).
The staff experiences described represented the forming and storming stages of team formation during the first year of the new service. These issues were identified as needing to be addressed prior to moving to the norming and performing team stages.

\subsection{Having a shared understanding of what characterises culturally safety in the local program context}

All staff interviewed expressed the importance of and personal commitment to cultural safety to ensure Aboriginal and Torres Strait Islander families were engaged in the program and received quality healthcare. Cultural safety was described by the interviewees as being responsive to the holistic needs of Aboriginal and Torres Strait Islander families:

we're trying to see what works better for our mums and that. And not only our mums, then we have our dads, and the grandparents and the aunties, that do come along and support their mums. So, you know, there are things that we are going to work on. If it's something that's not working, well then, you know, we put it out there and suggest to the mums, "Well what do you want? It's not all about us, it's what, it's for you." (Emily, Indigenous)

While there appeared to be understanding and desire amongst all staff for women to experience culturally safe care, it was acknowledged that culture was diverse and dynamic; hence, so were women's cultural needs. From the interviews, there did not appear to be consensus on how cultural safety was to be operationalised within this program nor was there initially a process to effectively identify and address concerns. Indeed, there were ambiguous interpretations about what was considered 'culturally safe' care. For example, one interviewee stated:

Arguing [something] in the name of culture, when you, when you unpack it and you go, "Oh actually, no, I'm pretty sure [that doesn't have] anything to do with culture ... . [We need] an honesty at times about culture being allowed to change and being different for different people at different times. (Robyn, Indigenous)

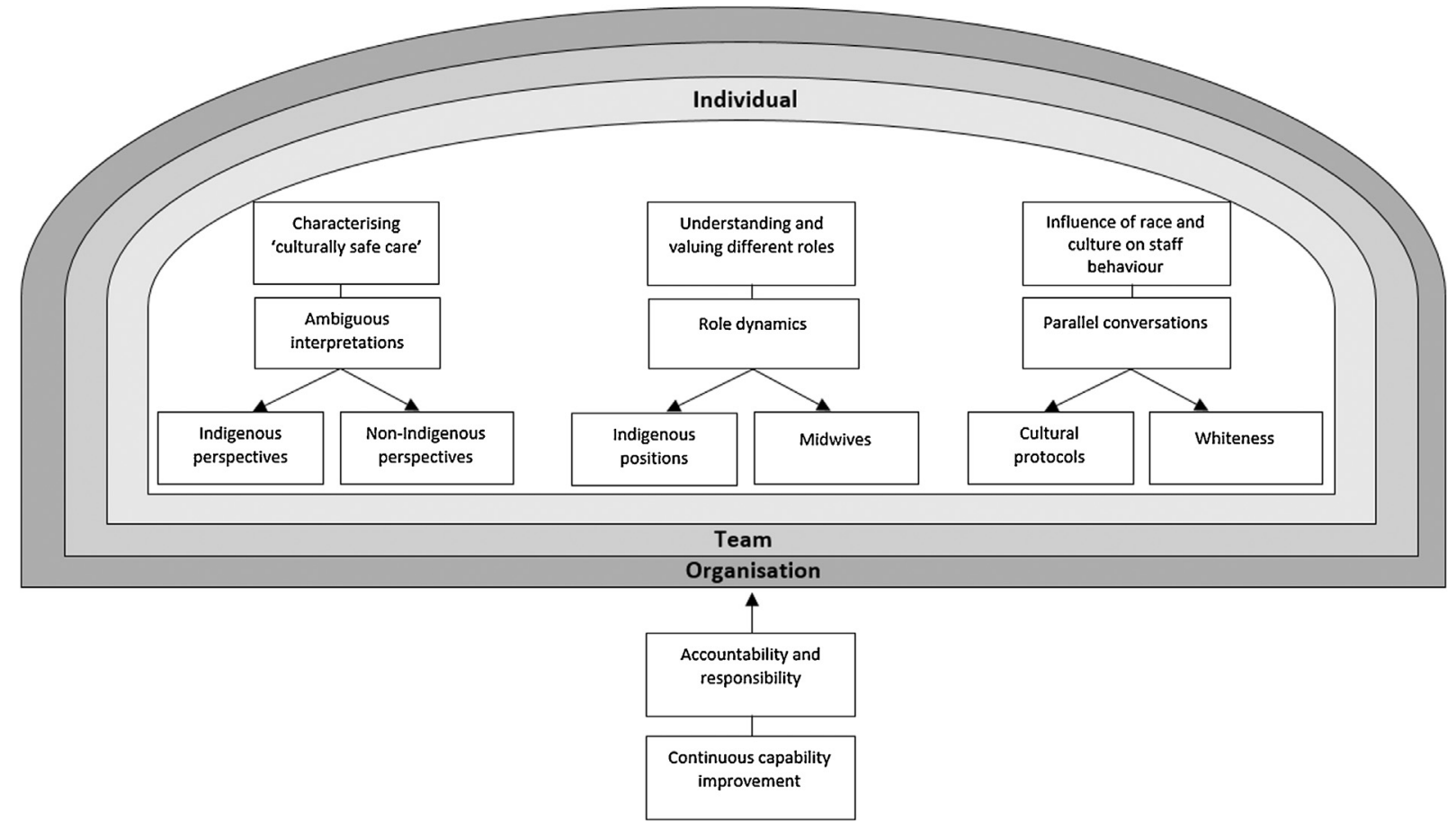

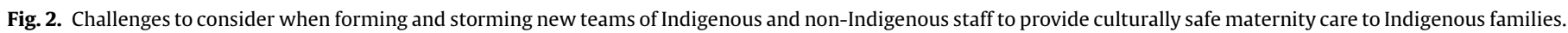


Non-Indigenous staff had a tendency to frame the accountability of being culturally safe practitioners to Indigenous staff as a disruption to their usual practice, a pressure or additional burden or task to their clinical role, highlighting the tensions in perceptions of behaviour:

Researcher: Have there been any challenges for you with that cultural side of providing the care?

Participant: Do you know what, there actually hasn't, not between me and the women. The actual issues come from, ... I suppose, [Indigenous staff] who say, "You don't know how to talk to our women." And I get really frustrated with that, because the women who I'm talking to are very diverse. Some of them are not even Indigenous, they have Indigenous partners. Or some of them have, you know, there's so many facets to, ah, what, what, what makes that person up. (Kate, non-Indigenous)

The Indigenous staff described providing culturally safe support and engaging families in the program as a key part of their job that they enjoyed and that came easily to them as it was part of their way of being and it was part of their cultural responsibility to look after others:

I grew up this way, I know what is best practice (Mandy, Indigenous)

The Indigenous staff described the pleasure and reward of doing this:

Every family's different. Um, they could be tough or they could be a breeze. [laughter] But yeah, you do have those ones that are tough, they're my priority, until ... I am satisfied and they have a smile on their face, so, yeah. I know that I'm doing my job right. (Emily, Indigenous)

4.2. Understanding and valuing different roles and knowledges people bring to the team

Indigenous and non-Indigenous staff, in management and service delivery, all described the importance of the Indigenous workforce in ensuring cultural safety and the need to increase the Indigenous presence within the program.

[The Indigenous clinician] brought tremendous cultural appropriateness of course by virtue that she was from the same group. (Linda, non-Indigenous)

Hiring Indigenous staff was sometimes assumed to be the panacea for acquiring a culturally safe workforce:

I think the employment of an Aboriginal midwife would be excellent ... it would overcome the problems. (Wendy, Indigenous).

However, for the staff currently working within the program, there appeared to be some confusion and some contention of the roles and responsibilities between the midwives and health workers about how they best work together.

For midwives who are very used to working in quite a one-to-one relationship with a woman and family. There's now another person in the mix and so working out exactly what [is] the purpose, the role, the value-add (Jenny, non-Indigenous)

The midwives didn't sort of see the importance of taking a health worker out. And it was a bit sad actually, [the health worker] was sort of stuck in the office. (Ellie, Indigenous)

A midwife explained they felt they were ultimately responsible for the women's care, ('At the end of the day the buck stops with us', Erica, non-Indigenous). This alludes to the dominance of Western medico-legal institutionalised responsibility, which at times was in contradiction with cultural responsibilities (i.e. cultural safety) which were as important to the Indigenous staff as clinical responsibilities (i.e. clinical safety) were to non-Indigenous staff.

One Indigenous staff member described an incident where she felt her role and expertise was dismissed by the midwife:

The midwives may not understand like health workers are health professionals and often, you know, people let us know things from the community, what might be happening with a situation, and with a client. And one, at one point a midwife sort of said to me, like, "Oh that's like community gossip." And I'm like, "Oh my God, it's not." It was one health professional telling me as a health professional that this is what she thought was needed, and that sort of opinion was a bit hurtful. But .. . that's how it works, but she's, the midwife is fantastic. Like culturally wise they're just, yeah when someone rings a health worker she doesn't see that as professional information. (Ellie, Indigenous)

From this excerpt, we can see Ellie valued what the midwife brought to the team in terms of clinical expertise and acknowledged the good intentions of the midwife and felt hurt when she felt the midwife did not acknowledge what the health worker brought to the team. There was tension expressed by Indigenous staff at this cultural interface, ${ }^{18}$ where Indigenous ways of doing became contested could be dismissed within the Western health system. This was reflected in the renumeration of roles with pay differentials between midwives and health workers.

They're not treatin' [the health worker as] your consultants, Indigenous consultants. They're the specialists in their field. (Carol, non-Indigenous)

Utilising the cultural knowledge and skills of the Indigenous staff was fundamental to achieving cultural safety within the program and has the potential to alleviate some of the perceived burden experienced by the midwives:

We need more of a team approach. [The midwives]'re thinking about the patient, I can see that. But they shouldn't try to do it all on their own. (Irene, Indigenous)

\subsection{Acknowledging the influence of race and culture on staff behaviour}

The influence of race and culture on staff behaviour appeared to be an important consideration amongst interviewees. However, from the interviews, it appeared that Indigenous and nonIndigenous staff did not commonly come together as a team to acknowledge and discuss race and culture. There were two parallel conversations that came to light from the interviews: (a) the impact of whiteness on the non-Indigenous staff; and (b) adhering to cultural protocol and Indigenous ways of knowing, being and doing for the Indigenous staff.

\subsubsection{Whiteness}

Whiteness manifested itself in the interviews via limited cultural understanding from the non-Indigenous staff who tended to frame Indigenous people and cultures as 'other'.

That whole cultural side of that and what that means, because to me that's a bit foreign. . . . and that's the hardest thing for me to navigate [laughs.] (Erica, non-Indigenous)

Belonging to the dominant culture, the non-Indigenous health staff were dismissive of the need to change or adapt when confronted with different world views or knowledges, categorising the change in practice as 'confusing':

when we do cultural safety days and things like that, I find that confusing. But if I just do what it is that I, you know, that I do and then no I don't find it confusing at all. (Kate, non-Indigenous) 
Indigenous staff working with the program felt there was limited understanding among the non-Indigenous staff about Indigenous ways of being, knowing, and doing, ${ }^{6}$ and how this may impact the care they provide.

[Non-Indigenous people are] not aware of it. . . . And sometimes it's body language. ... And body language says a lot when you're sitting [watching]. And [the Indigenous women] don't feel comfortable [but they will] say and do the right things ... [ [then] you can talk to a midwife, [who will say] "Oh God, I got a great rapport with that [woman], oh yeah, she's really good." And you think, "Not what I heard." (Irene, Indigenous)

This knowledge gap was seen to be independent of intent: I've always maintained that people only know what they know. (Mandy, Indigenous)

Addressing whiteness through critical reflection with midwives was seen to have challenges, both in the service and more generally:

[The midwives]'re working really hard, trying really hard ... others just aren't listening and so the, people have just shut up, they stop talking to them and that happens a lot... White midwives, don't take criticism very well. ... we have to be really gentle on these midwives or we're going to lose them. ... I think they all have good hearts, it's just that some, some are probably on the mark while others aren't. (Carol, non-Indigenous)

Whiteness was not perceived as an immutable trait for nonIndigenous people but as something that could be managed through self-reflection:

Even though [she] wasn't [Indigenous], had an idea of where things were coming, because she's, she's lived a life, so she knows. (Ellie, Indigenous)

\subsubsection{Cultural protocol}

For the Indigenous staff, cultural protocol - Indigenous ways of knowing, being and doing - also appeared to influence staff behaviour. Enacting cultural protocols is the manifestation of relationality i.e. the connectedness to people, to place, to ceremony and cultural practices; the interconnectedness of all elements of knowing, being and doing. ${ }^{6,47}$ Protocols are the mechanisms that define, regulate and enact behaviour between Indigenous people and others regardless of whether they are Indigenous or non-Indigenous. Some Indigenous staff discussed how they did not feel comfortable talking about certain cultural aspects or being expected to be the cultural expert on all things Indigenous, not wanting to break protocol or contribute to the discourse of homogenous pan-Aboriginality.

Researcher: Do the ... midwives draw on you for advice about cultural aspects of birth or providing the service?

Participant: No. They did at first when I was placed in, for that, but I think, ah, no.

Researcher: Would you be comfortable providing that type of role?

Participant: Only for my own culture, and from where I'm from, which is a bit hard, because it's [very different] we all got different rituals and customs ... I I would definitely not give any advice on a [Community] family with high social needs without consulting with one of the aunties. (Sarah, Indigenous)

Cultural protocol informed the mechanisms of cultural safety, with Indigenous staff being the knowers of appropriate protocols and norms of engagement. If the Indigenous staff were not comfortable or aware of something, they knew the culturally appropriate process to obtain that information. This skill appeared to be currently underutilised within the program. One suggestion was to design a new role specifically for a 'cultural mentor' within the program:
I've maintained all along that this programme needs somebody who's not the boss, but is able to pull all of these roles together in harmony. And be, also be able to offer that knowledge and expertise, and when I say that I mean cultural knowledge and cultural expertise, um, like a mentoring kind of role as well. (Mandy, Indigenous)

\subsection{Acting on individual and organisational responsibilities for continuous improvement towards cultural safety}

\subsubsection{Individuals as active learners}

A strong message from these interviews was the need to support non-Indigenous staff to build their cultural competencies; this was particularly emphasised by the Indigenous workforce. Given that this was early days of the new service, with some midwives who did not have previous experience working with Indigenous families, there was an expectation that individuals had to take responsibility for their own active learning. This is in a context where Aboriginal and Torres Strait Islander health and cultural safety education and training were not mandatory in undergraduate midwifery training until recently nor were they mandatory for ongoing professional development at the hospital.

Despite being an important means of self-education, it was iterated that learning about Aboriginal issues from books was not seen as sufficient: '[just] because you've read a book, it means nothing' (Irene, Indigenous). Linda, a non-Indigenous staff member not directly providing care to families within the service, said:

I actually was speaking to one of [the midwives] recently who said, "I need to talk to you more about cultural stuff because I've read this textbook and it's still all woolly, and-and, you know, I need to know how to do it." And I said, "Well, there's no 'it' love.". . . And [the midwife is] genuinely wanting to do it better.

While it was widely acknowledged that learning how to be a culturally safe clinician was important, a major question was how to best go about this as a non-Indigenous person.

This was seen as a long-term commitment and a personal journey:

I still learn a lot from sitting around a table with Aboriginal people, and I think that's, you know, it's lifelong learning. (Mary, nonIndigenous)

Charlotte (non-Indigenous) reflected on the importance of enabling non-Indigenous staff to endeavour to become culturally safe, as ultimately they are the ones responsible for their own learning and accountable for their own actions:

When are we ever going to build bridges if we're never allowed to start relationships on our own, you know? Um, and, and honestly, if we're not safe enough to be left alone with people in the community, like if we're culturally that inappropriate, we shouldn't be in the job. (Charlotte, non-Indigenous)

\subsubsection{Organisations as potential enablers of cultural safety}

Employing organisations were seen as both an enabler and barrier for promoting cultural safety: either as an active employer of Aboriginal and Torres Strait Islander people or through provision of professional development opportunities for staff aspiring to become culturally safe. At the time of the interviews, no mandatory cultural safety training was available to hospital staff. A specific two-day orientation (additional to hospital and mandatory orientation) was developed for the midwives entering the new maternity service and orientation at all three partner organisations was also added. Amongst interviewees, this cultural orientation did not appear to be sufficient:

The midwives should have had a cultural awareness course, ... . because yes clinically, they were fine. But as far as the cultural side of it, they weren't. (Wendy, Indigenous). 
Organisational support in promoting a culturally safe workforce was not seen as exclusive to providing training. The need for organisational cultural competency was acknowledged as this would remain a barrier to staff members' ability to be culturally safe:

Some of the midwives have had a bit of cultural sort of understanding, but they are also between a rock and a hard place in that they work within a system that is based on western medicine, and they have policies and procedures that have to be followed. (Carol, non-Indigenous)

ACCHOs were regarded as leaders for supporting and enabling staff endeavours towards cultural safety:

I see [the ACCHOs] as being the main player [in providing training]. And it has to be a cultural package, it has to have resources, so it's online, it's CD-ROM, but it's also that face-to-face stuff. ... I reckon a weekend workshop where we go on country with elders and they're actually, believe it or not, sitting around a camp, I know it sounds a strange, but sitting around a campfire and talking. Talking, talking, talking. (Bronwyn, Indigenous)

This experiential learning, coupled with critical reflective practice, ${ }^{20}$ was seen as more effective than book learning, with greater potential to value Indigenous knowledges as it is often how Indigenous people themselves learn about cultural protocols.

During the data collection period, one of the Indigenous partner organisations started providing cultural supervision to guide critical reflective practice for the non-Indigenous midwives. This included debriefing led by ACCHO Indigenous social health practitioners to increase cultural and clinical safety and reduce burnout of the midwives. This was described by the midwives in the interviews as a designated space for exploring issues of concern impacting their current practice.

We have been getting [cultural and] clinical supervision fortnightly, which is, that is just a godsend. (Kate, nonIndigenous)

As one interviewee explained:

[It] allowed, particularly in the early stages, a bit more honestly from the midwives themselves and, and, you know, about, "I don't know this stuff and I . . " Or, "I might sound racist," or, you know, exploration of that stuff without any fear of, um, pissing your coworkers off, effectively. Um, and so it has become a really safe space, for that reason ... The midwives are dragging themselves in when they've had two hours' sleep and, you know, should be on holidays (Robyn, Indigenous)

This initiative proved pivotal in deepening the critical and cultural understanding of the non-Indigenous staff. At the time of interview, cultural supervision was only available to the nonIndigenous staff yet there was demand among the Indigenous staff to be included or have a similar forum which was established later.

\section{Discussion}

This study identified some key challenges experienced by multidisciplinary, culturally diverse health staff and opportunities for workforce investment when forming and norming new teams aiming to provide culturally safe maternity care for Aboriginal and Torres Strait Islander families in a large Australian city. It is these frontline workers-predominantly midwives and Indigenous health workers-who must navigate and negotiate daily the complex terrains of the current Australian health system, often obstructionist in meeting the needs of Aboriginal and Torres Strait Islander people.

Fig. 2 summarises the learnings from the study and can provide discussion points for new teams as an iterative process that needs to occur regularly. It is by no means prescriptive or exhaustive and was grounded in this program's local context. The findings emphasise the importance of exploring and understanding Indigenous and non-Indigenous perspectives of culturally safe care, defining and valuing the responsibilities and roles of midwives and health workers and Indigenous knowledges of the Aboriginal and Torres Strait Islander staff, acknowledging the impact of whiteness and cultural protocol on staff behaviour, and both reflecting and acting on both individual and organisational responsibility to promoting a culturally safe workforce.

At the core of Western midwifery is the principle of working in partnership between midwife and woman. ${ }^{20}$ In our study, the new service design saw the core team extend to include a role for the Indigenous health worker, as requested by Aboriginal and Torres Strait Islander women and community stakeholders. ${ }^{37}$ The Indigenous health worker played an important role in ensuring the cultural safety of the service, but interviewees made it clear that cultural safety was also the responsibility of all staff. It was acknowledged that non-Indigenous staff needed to be self-reflective, understand their privilege influenced their clinical practices as well as being receptive to learning from their Indigenous colleagues. In these interviews, the power dynamic between midwife and health worker was apparent: the midwives framed midwifery care as taking precedence in this transaction with women, which was supported by institutionalised Western Scientific knowledge and responsibilities in the maternity health system and regulatory bodies and codes of practice. The Indigenous staff stressed the importance of cultural safety or cultural ways of 'doing' for the success of this program, and that community knowledge should be equally valued within the health system and community responsibilities and protocols also recognised.

Non-Indigenous staff reported the 'cultural dimension' of their work providing maternity care to Indigenous families as an additional pressure to their clinical care, unsure of how they should operate and what they should do; in contrast to the Indigenous staff who saw it as inherent to the work and instinctive. Growing the number of Indigenous clinicians was signalled as one way to increase cultural safety of clients, though being Indigenous did not make them immune to the challenges of working in a Western Scientific Knowledge dominated space nor guarantee their own cultural safety. To fully meet the comprehensive maternity needs of Aboriginal and Torres Strait Islander people, a health system needs to systemically incorporate Indigenous ways of knowing, being and doing to respond to a holistic definition of health ${ }^{1}$ and be 'free of racism' ${ }^{2}$ and its adverse effects. ${ }^{48}$ Some interviewees spoke about the need for non-Indigenous staff to recognise their privilege of belonging to the dominant group and argued they should incorporate, not dismiss, Indigenous ways of knowing, doing and being into service provision. Whilst on the whole non-Indigenous staff expressed a willingness to do this, they expressed uncertainty about how to do this. Further research is needed to understand how to implement this on a large scale.

Many health professionals and researchers in Australia refer to 'bicultural' or 'two-way' learning when working with Indigenous clients or Indigenous workers. ${ }^{34}$ Focusing simply on reciprocal learning (e.g. non-Indigenous midwives learning from Indigenous health workers, or vice versa) can silence or minimise the inequality of this relationship due to the continued marginalisation of Indigenous knowledges from the mainstream Australian health system. Indigenous workers may be cultural knowledge holders; however, they are disproportionately required to educate and advocate the legitimacy of their roles and Indigenous ways of knowing and doing, often in isolation or without systemic support to do so. ${ }^{17}$ A culturally safe health system can only prevail when there is institutional and operational support to do this and a broad understanding of how to do it. ${ }^{49}$ 


\subsection{Recommendations}

This study informed the early stages of a broader participatory action research project aimed at redesigning maternity services to improve health outcomes for Aboriginal and Torres Strait Islander mothers and babies. At time of writing, the maternity service continued to evolve, in response to identified staff, family, and community needs. Critical reflective practice provided a key mechanism for transitioning from the team forming and storming stages, aiming to progress towards cultural safety. Table 2 details the key actions undertaken through the participatory action research process to address key issues identified from the staff interviews. We believed the following recommendations would be applicable to other settings, all requiring a trusting and respectful context to be successful. Firstly, staff should have a personal ongoing commitment to critically self-reflect on their practice, an understanding of white privilege, racism and approach cultural safety as an endeavour that involves life-long learning. This should be embedded in staff recruitment processes and ongoing professional development activities. Teams should workshop a working definition or operationalisation of what cultural safety means within their local service context. The specific roles and contribution of Indigenous health workers to the team needs to be clear and valued by all staff, with explicit efforts to increase the numbers of Indigenous people working within the program. Teams should openly discuss and acknowledge the influence of race and culture on staff behaviour and value Indigenous knowledges in everyday program delivery. Finally, organisations need to systematically provide cultural safety training for all staff, consider cultural supervision for frontline staff, and have aligned organisational strategies, policies and procedures that promote a culturally safe environment for staff and clients. ${ }^{50}$ The need for group cultural supervision was identified by the ACCHOs as a way to support greater understanding and the team forming and storming processes, a mechanism to assist with forging the team to come together to discuss race, culture and clinical practice. All of these are integral first steps towards acknowledging the power imbalances between Aboriginal and Torres Strait Islander people and non-Indigenous people in the Australian maternity system. These recommendations strongly align with Australia's National Aboriginal and Torres Strait Islander Health Plan vision for a health system 'free of racism' by $2023 .^{2}$

Table 2

Recommendations to transition from forming and storming to norming and performing culturally safe teams.

Issues identified from staff interviews How we responded Suggestions going forward

Individual

Personal ongoing commitment for critical self-reflection and life-long learning

Team

No agreed working definition of what is culturally safe care within this service

Lack of clarity of roles

Limited team discussion about influence of race and culture on staff behaviour

\section{Organisational}

Few Indigenous staff providing frontline services

Limited cultural understanding of nonIndigenous staff

No cultural competency training for hospital staff

Limited policy and procedural support from the hospital to value Indigenous was of knowing and doing
This has been workshopped in team meetings and off-site workshops, developing agreed expectations of culturally safe service delivery (ongoing)

The Indigenous maternal infant health worker role was changed to a Family Support Worker and the job description was revised to better reflect the holistic, social, emotional, cultural and practical support they provide families. This included a pay rise and shifted employer from the hospital to the ACCHO so the Family Support Workers could access a greater peer network, integration with the primary healthcare setting and additional training. Information about the Family Support Worker role was also included in midwife orientation. This led to greater understanding from midwives about the role and value of Indigenous workers in the service.

Shared clinical and cultural supervision and 4 two-hour sessions (see below) provided by the ACCHO allowed Indigenous and non-Indigenous staff to openly discuss the influence of race and culture on their practice as a team.

The partnership maternity service specifically targeted the recruitment of Indigenous staff, increasing from 2.6 fulltime equivalent Indigenous staff members working in the service to 15 fulltime Indigenous staff members in the two years following the interviews. Identified positions include hospita liaison officers, Family Support Workers, student midwifery cadets, new graduate midwife position and Hub manager. ACCHO provides induction into their cultural integrity framework at initial staff orientation. ACCHO also provides ongoing clinical and cultural supervision to the whole team. In 2017, the staff undertook 4 two-hour sessions of critical reflection about Indigenous ways of knowing, being and doing, provided by two cultural advisors from the ACCHO. This focused on Aboriginal ways of knowing, being, seeing and doing through team reflection. Staff also attended off-site workshops including visiting an Aboriginal community to reflect on ongoing intergenerational trauma of historical government policies.

The ACCHO developed their cultural integrity framework. New study has commenced to describe, measure and improve organisational strategies to meet the needs of Aboriginal and Torres Strait Islander people at the hospital.
Embed in team documents such as orientation manual, posters in office, report templates as reminders. Periodically review in team meetings.

Openly value Indigenous knowledges - ways of knowing, doing, seeing and being.

Create identified clinical positions for Indigenous people within the hospital (e.g. midwifery graduate positions). Continue to provide mentorship and support for Indigenous staff, including career pathways as appropriate, cadetships for midwifery training.

Hospital to provide cultural competency training for all staff (not just staff employed in the Indigenous maternity service).
Cultural mentoring embedded into the program for both Indigenous and non-Indigenous staff. Hospital to develop organisational policies and procedures aimed to improve organisational cultural competency and meet the needs of Aboriginal and Torres Strait Islander people, including zero tolerance to racism. 


\subsection{Limitations}

Whilst interviewee demographics were representative of the body of staff, it is possible that staff who did not participate in this study may have had diverging views, hence this study is only a partial representation of the experiences of partnership staff. Staff may have been cautious in their responses; non-Indigenous staff may have avoided discussing contentious issues of race in favour of not wanting to appear racist. ${ }^{32}$ Lastly, in the absence of hearing directly from women, there remains ambiguity about the actual cultural safety of their healthcare experiences. Whilst not the focus of this present study, we are concurrently undertaking surveys and interviews with women to assess this in the broader participatory action research study. Early indications are positive as women are presenting early to book into the model and demand is such that it has never had to be advertised and has been expanded.

\section{Conclusion}

There is an urgent need for increased cultural safety of Australian health services to improve quality of care and health outcomes for Aboriginal and Torres Strait Islander people. This study has provided empirically-based, theory-informed recommendations for health service planners and providers to support staff in the initial stages of establishing a new partnership-based maternity service. All staff interviewed were committed to providing culturally safe care; workforce investment in the early stages of team formation can assist with the 'how' of doing this. The participatory action research process was important in identifying and responding to staff-identified opportunities for emancipatory change in becoming a culturally safe team. Despite the challenges presented from the first year of the new service, the team began to see improvements in clinical outcomes for the families for whom they were providing care. ${ }^{37}$ This supports our recommendation for conscientious workforce investment in the early stages of team formation that centres on culturally safe reflective practice will support learning and transition from cultural novice to cultural safety is vital to accelerating health gains for Aboriginal and Torres Strait Islander people.

Cultural safety is ultimately defined and assessed by the end user; this is a dynamic concept that requires transformative reflexive practice and genuine relationships and partnerships. ${ }^{20}$ Without challenging the systematic processes that favour imposed Western biomedical knowledges in the Australian maternity care system, staff working within this system will be limited in their capacity to enact cultural safe care for Aboriginal and Torres Strait Islander women and families; therefore, redesign that embeds Indigenous knowledge as evidence-based practice is vital. Intrapartum care remaining in the almost exclusive realm of Australian hospitals denies large-scale inclusion of Indigenous knowledges and self-determination required for culturally safe maternity care for Aboriginal and Torres Strait Islander people. Genuine hospital partnerships with Indigenous communities is one step towards addressing this. ${ }^{49}$

\section{Role of funding source}

Funding for this study came from the partner organisations and an National Health and Medical Research Council Partnership Grant (APP1077036). The National Health and Medical Research Council did not have any role in the study design, data collection, data analysis, data interpretation, or writing of this manuscript.

\section{Ethical statement}

Ethical approval for this study was granted by the hospital organisation's Human Research Ethics Committee (HREC/14/MHS/ 168) on 4 November 2014.

\section{Author contributions}

SKi, KC and YR conceived the initial study and interview guide. KC drafted and submitted the Research Ethics and Governance applications, recruited and consented participants and conducted the interviews. SH led the manuscript writing and data analysis, and coded the data. YR provided Indigenous oversight to the study, supported by KW-I, RW, RB and SW, and assisted SH with interpretation of the data and refining of themes. KW-I, RW, SKr, RB and SW assisted with the refining of the final themes and provided Indigenous perspectives and/or industry/program knowledge to ensure relevance of recommendations to the study context. All authors assisted with editing and finalising the manuscript.

\section{Conflicts of interests}

None declared.

\section{Acknowledgements}

The authors would like to thank Dr Helen Stapleton, Ms Machellee Kosiak and Dr Carmel Nelson for their assistance at various points of the study. We would also like to acknowledge the other study investigators and participants, as well as the three partner organisations.

\section{References}

1. National Aboriginal Health Strategy Working Party. A National Aboriginal Health Strategy. Canberra: NAHSWP; 1989.

2. Commonwealth of Australia. National Aboriginal and Torres Strait Islander Health Plan (2013-2033). Canberra: Australian Government; 2013.

3. Australian Health Ministers' Advisory Council. Aboriginal and Torres Strait Health Performance Framework 2017 report. Canberra: AHMAC; 2017.

4. Clifford A, McCalman J, Bainbridge R, Tsey K. Interventions to improve cultural competency in health care for Indigenous peoples of Australia, New Zealand, Canada and the USA: a systematic review. Int J Qual Health Care 2015;27(2):8998.

5. Alford K. A cost-effective approach to' Closing the Gap' in health, education and employment: investing in Aboriginal and Torres Strait Islander nursing education, training and employment. Canberra: Congress of Aboriginal and Torres Strait Islander Nurses and Midwives; 2015.

6. Martin K. Ways of knowing, being and doing: a theoretical framework and methods for Indigenous and Indigenist re-search. J Aust Stud 2003;27 (76):203-14.

7. Sherwood J, Edwards T. Decolonisation: a critical step for improving Aboriginal health. Contemp Nurse 2006;22(2):178-90.

8. Australian Institute of Health and Welfare. Australia's mothers and babies 2016in brief. Perinatal statistics series no. 34. Cat. no. PER 97. Canberra: AIHW; 2018.

9. Kildea S, Tracy S, Sherwood J, Magick-Dennis F, Barclay L. Improving maternity services for Indigenous women in Australia: moving from policy to practice. Med J Aust 2016;205(8):374-9.

10. Kildea S, Van Wagner V. 'Birthing on Country' maternity service delivery models: a review of the literature. Canberra: Maternity Services Inter-Jurisdictional Committee for the Australian Health Minister's Advisory Council; 2013.

11. Kildea S, Magick-Dennis F, Stapleton H. Birthing on Country workshop report, Alice Springs, 4th July. Brisbane: Maternity Services Inter-Jurisdictional Committee; 2012

12. Ramsden I. Cultural safety and nursing education in Aotearoa and Te Waipounamu. Wellington: Victoria University of Wellington; 2002.

13. Campinha-Bacote J. Cultural competence in psychiatric mental health nursing. A conceptual model. Nurs Clin North Am 1994;29(1):1-8.

14. Tuckman BW. Developmental sequence in small groups. Psychol Bull 1965;63:384-99.

15. Tuckman BW, Jensen MAC. Stages of small-group development revisited. Group Organ Manage 1977;2(4):419-27.

16. West R, Gamble J, Kelly J, Milne T, Duffy E, Sidebotham M. Cultural capable and cultural safe: caseload care for Indigenous women by Indigenous midwifery students. Women Birth 2016;29:524-30. 
17. C. Bond, M. Brough, J. Willis, J. Stajic, B. Mukandi, C. Canuto, et al., Beyond the pipeline: a critique of the discourse surrounding the development of an Indigenous primary health care workforce in Australia. Aust J Prim Health (in press).

18. Nakata M. The cultural interface. Disciplining the savages, savaging the disciplines. Canberra: Aboriginal Studies Press; 2007.

19. Australian Commission on Safety and Quality in Healthcare. National Safety and Quality Health Service Standards. 2nd edn. Sydney: ACSQHC; 2017.

20. Nursing and Midwifery Board of Australia. Midwifery standards for practice. Melbourne: Nursing and Midwifery Board of Australia; 2018.

21. Nursing and Midwifery Board of Australia. Code of conduct for midwives. Melbourne: Nursing and Midwifery Board of Australia; 2018.

22. Paradies Y, Truong M, Priest N. A systematic review of the extent and measurement of healthcare provider racism. J Gen Intern Med 2014;29 (2):364-87.

23. Durey A. Reducing racism in Aboriginal health care in Australia: where does cultural education fit? Aust N Z J Public Health 2010;34(Suppl. 1):S87-92.

24. Liaw, ST, Wade, V, Furler JS, Hasan I, Lau P, Kelaher M, etal. Cultural respect in general practice: a cluster randomised controlled trial. Med J Aust 2019;210(6):263-8.

25. Nguyen HT, Gardiner A. Indigenous community members as teachers of Indigenous health. Aust Fam Physician 2008;37(12):1019-21.

26. Abbott P, Gordon E, Davison J. Expanding roles of Aboriginal health workers in the primary care setting: seeking recognition. Contemp Nurse 2007;26(1):66-73.

27. Stamp G, Champion S, Anderson G, Warren B, Stuart-Butler D, Doolan J, et al Aboriginal maternal and infant care workers: partners in caring for Aboriginal mothers and babies. Rural Remote Health 2008:8(3):883.

28. Kirkham R, Rumbold A, Hoon W, Stuart-Butler D, Moore V. Emotional labour and Aboriginal maternal infant care workers: the invisible load. Women Birth 2018;31(2):110-6.

29. Kowal E, Franklin H, Paralikar V. Reflexive antiracism: a novel approach to diversity training. Ethnicities 2013;13(3):316-37.

30. Downing R, Kowal E. A postcolonial analysis of Indigenous cultural awareness training for health workers. Health Sociol Rev 2011;20(1):5-15.

31. McIntosh P. White privilege: unpacking the invisible knapsack. Philadelphia: Independent School; 1990.

32. DiAngelo R. White fragility. Int J Crit Pedagogy 2011;3(3):54-70.

33. Paradies Y. Defining, conceptualizing and characterizing racism in health research. Crit Public Health 2006;16(2):143-57.

34. Eckerman A-K, Dowd T, Chong E, Nixon L, Gray R, Johnson SM. Binan Goonj: bridging cultures in Aboriginal health. 3rd edn. Chatswood: Churchill Livingstone, Elsevier; 2010

35. Hickey S, Roe Y, Gao Y, Nelson C, Carson A, Currie J, et al. The Indigenous Birthing in an Urban Setting study: the IBUS study. BMC Pregnancy Childbirth 2018;18:431-43.
36. Markham F, Biddle N. Recent changes to the Indigenous population geography of Australia: evidence from the 2016 Census. Aust Popul Stud 2018;2(1):1-13.

37. Kildea S, Hickey S, Nelson C, Currie J, Carson A, Reynolds M, et al. Birthing on Country (in Our Community): a case study of engaging stakeholders and developing a best-practice Indigenous maternity service in an urban setting. Aust Health Rev 2018;42(2):230-8.

38. Smith L, Rosenzweig L, Schmidt M. Best practices in the reporting of participatory action research: embracing both the forest and the trees. Couns Psychol 2010;38(8):1115-38.

39. Brydon-Miller M. Participatory action research: psychology and social change. J Soc Issues 1997;197(53):657-66.

40. Hickey SD, Maidment S-J, Heinemann KM, Roe YL, Kildea SV. Participatory action research opens doors: mentoring Indigenous researchers to improve midwifery in urban Australia. Women Birth 2018;31(4):263-8.

41. National Health and Medical Research Council. Values and ethics: guidelines for ethical conduct in Aboriginal and Torres Strait Islander health research. Canberra: National Health and Medical Research Council; 2003.

42. National Health and Medical Research Council. Keeping research on track: a guide for Aboriginal and Torres Strait Islander people about about health research ethics. Canberra: National Health and Medical Research Council; 2005.

43. Mason J. Semistructured interview. In: Lewis-Beck MS, Bryman A, Liao TF, editors. The SAGE encyclopedia of social science research methods. Thousand Oaks: SAGE Publications Inc.; 2004. p. 1021.

44. Frey J. Group interviews. In: Lewis-Beck MS, Bryman A, Liao TF, editors. The SAGE encyclopedia of social science research methods. Thousand Oaks: SAGE Publications, Inc.; 2004. p. 445.

45. Pezalla A, Pettigrew J, Miller-Day M. Researching the researcher-as-instrument: an exercise in interviewer self-reflexivity. Oual Res 2012:12(2):165-85.

46. Braun V, Clarke V. Using thematic analysis in psychology. Qual Res Psychol 2006;3(2):77-101.

47. Graham M. Some thoughts about the philosophical underpinnings of Aboriginal worldviews. Worldviews Glob Religions Cult Ecol 1999;3(2):105-18.

48. Kelaher MA, Ferdinand AS, Paradies Y. Experiencing racism in health care: the mental health impacts for Victorian Aboriginal communities. Med J Aust 2014;201(1):44-7.

49. Burton J. Opening doors through partnerships: practical approaches to developing genuine partnerships that address Aboriginal and Torres Strait Islander community needs. North Fitzroy: Secretariat of National Aboriginal and Islander Child Care; 2012.

50. Coffin J. Rising to the challenge in Aboriginal health by creating cultural security. Aboriginal Islander Health Worker J 2007;31(3):22-4. 